\title{
Docking Applied to the Study of Inhibitors of c-Met Kinase
}

\author{
Luciana Gavernet, Pablo Hernan Palestro, and Luis Bruno-Blanch \\ Medicinal Chemistry, Department of Biological Sciences, Faculty of Exact Sciences, National University of La Plata, \\ 47 and 115, La Plata B1900BJW, Argentina
}

Correspondence should be addressed to Luciana Gavernet, lgavernet@biol.unlp.edu.ar

Received 8 September 2011; Accepted 22 September 2011

Academic Editors: B.-T. Liu and A. Liwo

Copyright () 2012 Luciana Gavernet et al. This is an open access article distributed under the Creative Commons Attribution License, which permits unrestricted use, distribution, and reproduction in any medium, provided the original work is properly cited.

Quinoxaline derivatives were studied as inhibitors of c-Met kinase, a receptor associated with high tumor grade and poor prognosis in a number of human cancers. In this paper we used docking methodologies to predict the binding conformation of a set of quinoxalines and to explain the differences of biological activities previously reported.

\section{Introduction}

Quinoxalines and quinoxalinones are attractive chemical candidates in medicinal chemistry due to their capacity to generate biological responses in their interaction with several biological targets. They show antiviral [1], herbicidal [2], and anti-inflammatory action [3].

Recent investigations revealed the pharmacological potential of quinoxalines as anticancer agents [4], and numerous theoretical studies were performed on quinoxaline and its derivatives, in order to find new antineoplastic compounds. Only a small number of them were based on molecular docking analyses, considering promising cancer targets from which structural information of the receptor is available. One example is the molecular docking of quinoxalines against human dihydrofolate reductase (hDHFR) performed by Corona and coworkers [5], with the aim of predicting the binding mode of quinoxalines in hDHFR. They used AutoDock 3.05 docking program, and the protein structure was taken from the crystal structure of the ternary complex hDHFR-NADPH-methotrexate [5].

Another recent investigation pointed out the quinoxaline scaffold as a template to the design of inhibitors of c-Met kinase [6]. This tyrosine kinase receptor is known to be deregulated and associated with high tumor grade and poor prognosis in a number of human cancers. In the course of one high throughput screening campaign, Porter and collaborators selected one quinoxaline derivative as the most promising structure to inhibit selectively the ATP binding site of $\mathrm{c}$-Met $\left(\mathrm{IC}_{50}=1.3 \mu \mathrm{M}\right.$ in the c-Met biochemical assay) [6]. After that, they successfully optimized the activity of the original structure by preparing and testing a set of quinoxalines. Another important contribution from this investigation was the X-ray crystal structure of the c-Met with one of the new inhibitors synthesized (pdb code 3F66 [7]).

Following Porter's research, we decided to contribute in the analysis of the interaction of quinoxalines with c-Met kinase. To this end, we used docking to predict the binding conformation of the entire set of inhibitors (Figure 1) and provided a rational explanation of the differences of activity in terms of the differences of the interaction with the receptor for the quinoxalines from which X-ray structures are not available.

\section{Materials and Methods}

2.1. Biological Data. The $\mathrm{IC}_{50}$ values for inhibitors of c-Met, that is, the concentration $(\mu \mathrm{M})$ of inhibitor that produces $50 \%$ inhibition of c-Met, were taken from the literature [6]. The reported biological evaluation was carried out with an IMAP time resolved fluorescence resonance energy transfer (TR-FRET) assay. Accordingly, the $\mathrm{pIC}_{50}\left(-\log \left[\mathrm{IC}_{50}\right]\right)$ are reported in Table 1. 
<smiles></smiles><smiles>CC(=O)Nc1cnc2cc(C(F)(F)F)cc(-c3ccccc3)c2n1</smiles><smiles></smiles><smiles>[R]c1cc(C(F)(F)F)cc2ncc(N3CCN(C)CC3)nc12</smiles><smiles>[R]Cc1ccc(OC)c(OC)c1</smiles>

13<smiles></smiles>

18<smiles>[R]=CNc1ccccc1</smiles>

23<smiles>[R]=CNCc1ccccc1[N+](=O)[O-]</smiles>

28<smiles>[R]=NCc1cccc(C(N)=O)c1</smiles>

32

14

19

24

29<smiles></smiles><smiles>[R]Cc1cccc([N+](=O)[O-])c1</smiles><smiles>[R]=NCc1ccc([N+](=O)[O-])cc1</smiles><smiles></smiles>

25<smiles>[R]=CC1=NON[C@@H]1C=C</smiles>

33<smiles>[R]=CNCCc1ccccc1</smiles>

26

27
30<smiles>[R]=NCc1ccc2nonc2c1</smiles>

34

Figure 1: Compounds selected for the docking analysis. 
TABLE 1: Biological data and docking results for quinoxaline derivatives.

\begin{tabular}{|c|c|c|c|c|c|c|c|c|c|}
\hline Compound & $\begin{array}{c}\text { c-Met } \mathrm{IC}_{50} \\
(\mu \mathrm{M})\end{array}$ & $\mathrm{pIC}_{50}$ & $d^{\mathrm{a}}(\AA)$ & $\begin{array}{c}\text { Binding energy } \\
(\mathrm{Kcal} / \mathrm{mol})\end{array}$ & Compound & $\begin{array}{c}\text { c-Met } \mathrm{IC}_{50} \\
(\mu \mathrm{M})\end{array}$ & $\mathrm{pIC}_{50}$ & $d^{\mathrm{a}}(\AA)$ & $\begin{array}{c}\text { Binding energy } \\
(\mathrm{Kcal} / \mathrm{mol})\end{array}$ \\
\hline 1 & 1.3 & 5.886 & 1.80 & -11.6 & 18 & 0.8 & 6.097 & 1.93 & -11.9 \\
\hline 2 & 0\%@100 & $<4$ & 1.92 & -10.5 & 19 & 12.9 & 4.889 & 2.24 & -10.5 \\
\hline 3 & 20.1 & 4.697 & 1.85 & -10.5 & 20 & 2.0 & 5.699 & 2.08 & -11.5 \\
\hline 4 & 5.3 & 5.276 & 1.78 & -11.3 & 21 & 0.39 & 6.409 & 2.09 & -12.0 \\
\hline 5 & 0\%@100 & $<4$ & 2.18 & -10.5 & 22 & 0.17 & 6.770 & 1.94 & -12.0 \\
\hline 6 & 1.3 & 5.886 & 1.86 & -11.7 & 23 & 0.33 & 6.481 & 1.92 & -12.2 \\
\hline 7 & 19.6 & 4.708 & 2.34 & -10.7 & 24 & 1.9 & 5.721 & 1.94 & -11.7 \\
\hline 8 & 28.3 & 4.548 & 2.03 & -10.6 & 25 & 1.1 & 5.959 & 2.07 & -11.7 \\
\hline 9 & 3.9 & 5.409 & 1.98 & -11.2 & 26 & 7.1 & 5.149 & 2.78 & -11.4 \\
\hline 10 & 2.8 & 5.553 & 1.94 & -11.4 & 27 & 0.035 & 7.456 & 1.86 & -12.4 \\
\hline 11 & 5.0 & 5.301 & 1.86 & -11.4 & 28 & 0.54 & 6.268 & 2.02 & -11.9 \\
\hline 12 & 60.2 & 4.220 & 3.20 & -10.7 & 29 & 0.055 & 7.260 & 1.97 & -12.0 \\
\hline 13 & 1.5 & 5.824 & 2.19 & -11.2 & 30 & 0.32 & 6.495 & 2.03 & -11.8 \\
\hline 14 & 1.8 & 5.745 & 2.05 & -11.6 & 31 & 0.017 & 7.770 & 1.96 & -13.0 \\
\hline 15 & 0\%@100 & $<4$ & 3.33 & -10.9 & 32 & 0.73 & 6.137 & 1.94 & -11.4 \\
\hline 16 & 5.0 & 5.301 & 3.35 & -11.1 & 33 & 0.031 & 7.509 & 2.02 & -12.4 \\
\hline 17 & 0.9 & 6.046 & 2.09 & -11.4 & 34 & 0.38 & 6.420 & 2.13 & -12.1 \\
\hline
\end{tabular}

${ }^{\mathrm{a}}$ Distances derived from docking between the endocyclic $\mathrm{N}$ atom located at the positions 4 of the quinoxalines and the $\mathrm{H}$ atom of the MET1160.

2.2. Docking. We used the automated docking program AutoDock 4.0 [8] to analyze the binding mode of the compound of the set and AutoDockTools 1.5.2 related software to prepare the inputs and analyze the results. The c-Met receptor was prepared from the $1.4 \AA$ resolution crystal deposited by Porter et al. (pdb code 3F66) [6]. The crystallographic water molecules, the original ligand (compound 17 , Figure 1), and the $\gamma$-butyrolactone crystallized from the buffer solution were stripped. Hydrogen atoms were added using the leap module of AMBER10 [9].

We used AutoDock force field $[10,11]$ and the default AutoDock parameters for all the variables. Gasteiger charges were calculated for the ligands and the receptor. Quinoxalines were docked considering two different possible protonation states: the neutral structure and one monoprotonated form (obtained by adding a hydrogen atom to the N4 of the molecules). In this way we explored the possibility of binding with protonated structures.

The ligands were docked using the Lamarckian Genetic Algorithm (LGA) in the active site region. This region was defined through a grid map with 60,60 , and 60 grid points $(X, Y, Z$ dimensions, resp.) that completely covers the space initially occupied by the crystallographic ligand (coordinates: $X=-0.013, Y=-23.028, Z=28.19)$. We use the default grid spacing ( 0.375 angstroms) and achieved 100 docking runs considering the quinoxalines as flexible, that is, all nonring torsions were considered active. The active site was considered as a rigid molecule for all the residues but MET1229 and TYR1230. It has been shown that these residues have different conformations in several crystal structures (cf., e.g., the pdb crystal structure of 1 ROP and 3F66), and these conformational changes have been considered in this investigation through flexible docking.

\section{Results and Discussion}

Table 1 shows the binding energies predicted by the AutoDock in conjunction with the biological data compiled from the literature. We report here the results obtained for the neutral form of quinoxalines, since no good results were found with the protonated form: the predicted binding energies were higher than those obtained from the neutral structures and they did not correlate with the experimental data.

A different situation was found with the neutral quinoxalines: AutoDock is capable of predicting the active conformation of compound 17, with RMS fit error of 0.158 relative to the crystallographic structures. Figure 2 shows the docked conformation of $\mathbf{1 7}$ in the active site. The binding reproduces the X-ray determined interaction between the N-4 atom of the quinoxaline ring and Met1160 of the kinase. These results increase our confidence in the predicted binding disposition of the other compounds, from which structural data is not experimentally available. Binding energies predicted by docking evidence an acceptable correlation with the biological data (Figure 3), that is, the most active compounds are those with more negative binding energy. The binding modes obtained by docking for the quinoxalines resemble the binding conformation determined for $\mathbf{1 7}$ by crystal structure data: the aromatic 8 -substituent is placed near the aromatic group of TYR1230 residue; the group is placed at the position 2 (generally the piperazine scalffold) points outside the cavity and is exposed to the solvent (Figure 4). Additionally, there are $\mathrm{H}$ bond interactions comprising the endocyclic $\mathrm{N}$ atom located at the position 4 of the backbone and one $\mathrm{H}$ atom of the MET1160. The distances between these 2 atoms are given in Table 1 for each molecule. The importance of 


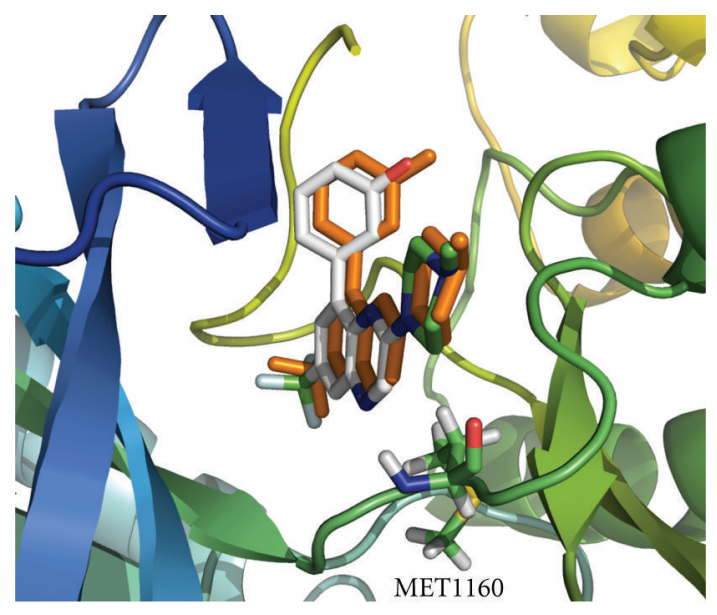

Figure 2: Superposition of the docking conformation (in white) and the crystallographic ligand (in orange) in the active site. Hydrogen atoms were omitted for simplicity.

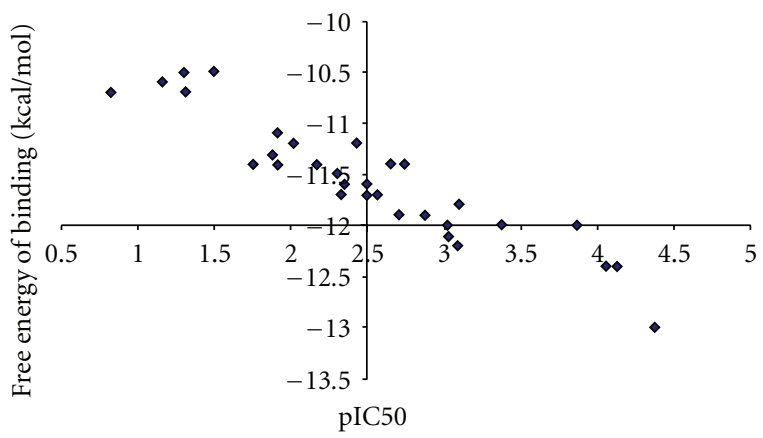

FIGURE 3: Correlation between the free energy of binding and biological data, expressed as $\mathrm{pIC}_{50}\left(r^{2}=0.865\right)$.

this $\mathrm{H}$-bonding interaction could be evidenced in the poor results found for the docking simulations for the protonated quinoxalines. In these structures, the H-binding was abolished and the results of docking were not satisfactory. In addition, we have to keep in mind the limitations of the calculations performed here. AutoDock force field estimates the desolvation potential based on the volume of atoms that surround a given atom and shelter it from solvent, so it does not consider the solvent explicitly [11]. This approximation might also be the cause of poor results in case of protonated ligands.

According to the data collected in Table 1, the inhibitory activity was improved by the inclusion of groups containing halogens at the position 6 of the quinoxaline skeleton since compound $\mathbf{8}$ is less active than compounds $\mathbf{1}, \mathbf{9}$, and 10. Docking conformations predict this increase through the better binding energy values, mainly caused by the positive interactions between halogens and the hydrophobic pocket placed in the active site, composed by lipophilic residues as VAL1092, LEU1140, LEU1157, and ALA1226 (Figure 4). This interaction was also observed in the binding conformation found for compound 11, which presents a

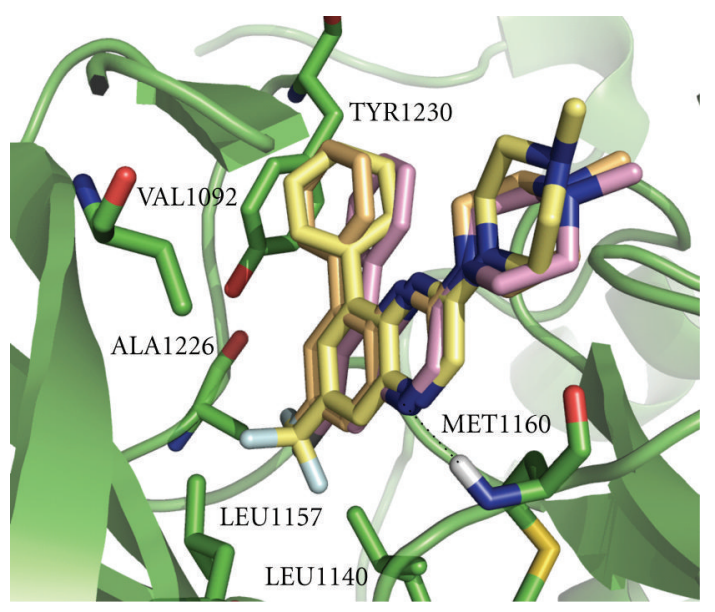

FIGURE 4: Docking solutions of the compounds 1 (carbon atoms in yellow), $\mathbf{9}$ (carbon atoms in orange), and $\mathbf{1 0}$ (carbon atoms in pink). Important residues of the active site of the kinase were highlighted (in green). Color code for heteroatoms is as follows: fluorine: cyan, nitrogen: blue, sulfur: yellow, oxygen: red, and chlorine: grey. Only one hydrogen atom of MET1160 was shown for simplicity. $\mathrm{H}$ bonding interactions between the molecules and this residue were shown as a dashed line. Distance values are given in Table 1.

methyl substituent, but not for compound 12 that has a polar cyan group at the 6 positions.

In order to maximize the $\pi$-interactions with the TYR1230, Porter et al. incorporated one larger link between the quinoxaline scaffold and the phenyl group (compounds 24-32, Figure 1) [6]. The best inhibitors obtained after this modification present a two-atom N-linking chain, with a 3-nitrosubstituent in the aromatic ring (compounds 27 and 31). Docking predicts the parallel-displaced stacking arrangement between the aromatic ring of TYR 1230 and the phenyl substituent of compounds 27 and 31 (Figure 5).

In agreement with other authors [6], we believe that the presence of the nitrogroup enhances the $\pi$-stacking interaction for the compounds by means of its electron-withdrawing effect. Additionally, we consider that the presence of the nitrogroup in metaposition is optimal for the interaction with ASP1222 through a hydrogen bond (Figure 5). The replacement of the amino methyl linker by sulfonamide group increases the activity (compound 31 ), possibly due to the capacity of sulfonyl group to interact with hydrophobic groups (Figure 5).

\section{Conclusions}

Docking techniques attempt to simulate the protein-ligand recognition through molecular modeling tools in a reasonable computation time. This objective represents a very ambitious task due to the complexity of the system: we need to simulate the interaction forces acting on the ligand and the receptor for each possible conformation of the two molecules, which means the consideration of hundred of 


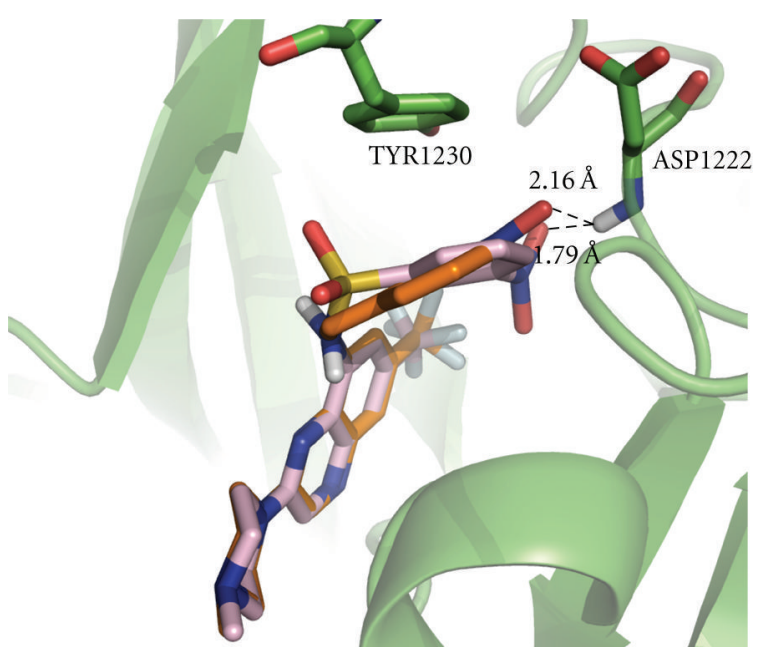

Figure 5: Top view of the active site of the kinase with the docking solutions for compounds 27 (carbon atoms in orange) and 31 (carbon atoms in pink). Distances between $\mathrm{O}$ atoms of the ligands and $\mathrm{N}$ atom of ASP1222 were measured for both compounds. Color code for heteroatoms is as follows: fluorine: cyan, nitrogen: blue, sulfur: yellow, and oxygen: red. Only one hydrogen atom of MET1160 was shown for simplicity.

thousands of degrees of freedom to explore the conformational space. For that reason these methodologies make simplifying assumptions to allow for reasonable computation time (such as restraining the movement of the protein and simplifying the mathematical expression of the forces acting on the complex), and these assumptions can lead to unusable results. Consequently, the results obtained through docking methodologies have to be considered keeping in mind these limitations.

Here, we report the results of applying one docking protocol to a set of quinoxalines and c-Met kinase in order to analyze the difference of activity. Although the analysis has to be considered preliminary, it has helped us to understand the trend in the inhibitory activity of this set. The docking results corroborate the experimental conformation found for one of the ligands (compound 17), correlate with the $\mathrm{IC}_{50}$ data, and allow us to analyze the importance of specific interaction for describing the affinity of different quinoxaline derivatives. These results can be considered only valid for the studied series, but for this case we provide a simple and useful tool to explain the interactions between the possible inhibitors and the receptor when the crystal structures of the complex are not available.

\section{Acknowledgments}

L. Bruno-Blanch is a member of the Facultad de Ciencias Exactas, Universidad Nacional de La Plata, P. H. Palestro is a fellowship holder of Consejo Nacional de Investigaciones Científicas y Técnicas de la República Argentina (CONICET) and L. Gavernet is a member of CONICET. This research was supported by the Agencia de Promoción Científica y Tecnológica (PICT 00339/2007), CONICET, and Universidad Nacional de La Plata, Argentina.

\section{References}

[1] F. Rong, S. Chow, S. Yan, G. Larson, Z. Hong, and J. Wu, "Structure-activity relationship (SAR) studies of quinoxalines as novel HCV NS5B RNA-dependent RNA polymerase inhibitors," Bioorganic \& Medicinal Chemistry Letters, vol. 17, no. 6, pp. 1663-1666, 2007.

[2] B. Li, H. Wu, D. Cui, D. Xiang, L. Bai, and H. Yang, "Unsaturated carboxylates and the use as herbicidide," WO Patent 125,337, November, 2006.

[3] A. Burguete, E. Pontiki, D. Hadjipavlou-Litina et al., "Synthesis and anti-inflammatory/antioxidant activities of some new ring substituted 3-phenyl-1-(1,4-di-N-oxide quinoxalin2-yl)-2-propen-1-one derivatives and of their 4,5-dihydro(1H)-pyrazole analogues," Bioorganic \& Medicinal Chemistry Letters, vol. 17, no. 23, pp. 6439-6443, 2007.

[4] A. Talevi, L. Gavernet, G. Serra, and L. E. Bruno Blanch, "Theoretic studies on quinoxaline and quinoxaline derivatives antineoplastic compounds," in QSAR Studies and Electronic Structure Methods Assisting the Design of Quinoxaline Drugs, Nova Science, 2010.

[5] P. Corona, M. Loriga, M. P. Costi, S. Ferrari, and G. Paglietti, "Synthesis of $N$-(5,7-diamino-3-phenyl-quinoxalin-2-yl)3,4,5-substituted anilines and $N$-[4[(5,7-diamino-3-phenylquinoxalin2-yl)amino]benzoyl]-l-glutamic acid diethyl ester: evaluation of in vitro anti-cancer and anti-folate activities," European Journal of Medicinal Chemistry, vol. 43, no. 1, pp. 189-203, 2008.

[6] J. Porter, S. Lumb, F. Lecomte et al., "Discovery of a novel series of quinoxalines as inhibitors of c-Met kinase," Bioorganic \& Medicinal Chemistry Letters, vol. 19, no. 2, pp. 397-400, 2009.

[7] H. M. Berman, J. Westbrook, Z. Feng et al., "The protein data bank," Nucleic Acids Research, vol. 28, no. 1, pp. 235-242, 2000, http://www.pdb.org/.

[8] G. M. Morris, D. S. Goodsell, R. S. Halliday et al., "Automated docking using a Lamarckian genetic algorithm and an empirical binding free energy function," Journal of Computational Chemistry, vol. 19, no. 14, pp. 1639-1662, 1998.

[9] D. A. Case, T. A. Darden, I. T. E. Cheatham et al., AMBER 10, University of California, San Francisco, Calif, USA, 2008.

[10] R. Huey, D. S. Goodsell, G. M. Morris, and A. J. Olson, "Gridbased hydrogen bond potentials with improved directionality," Letters in Drug Design and Discovery, vol. 1, no. 2, pp. 178-183, 2004.

[11] R. Huey, G. M. Morris, A. J. Olson, and D. S. Goodsell, "A semiempirical free energy force field with charge-based desolvation," Journal of Computational Chemistry, vol. 28, no. 6, pp. 1145-1152, 2007. 


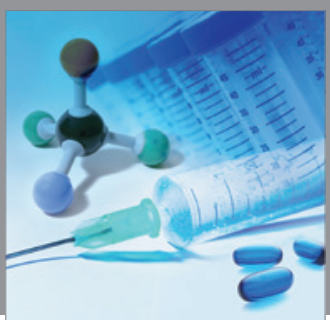

International Journal of

Medicinal Chemistry

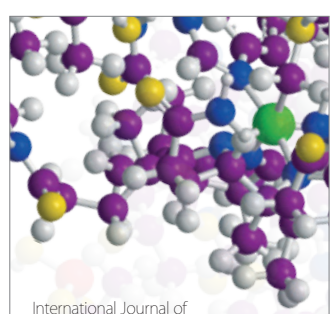

Carbohydrate Chemistry

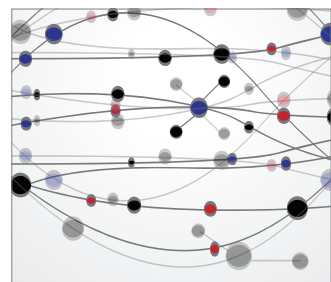

The Scientific World Journal
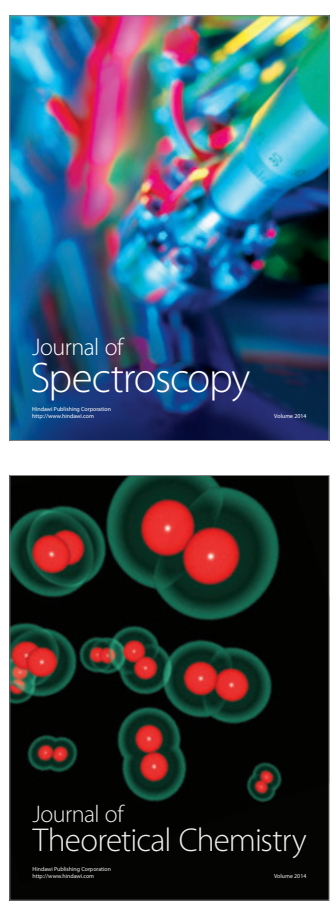
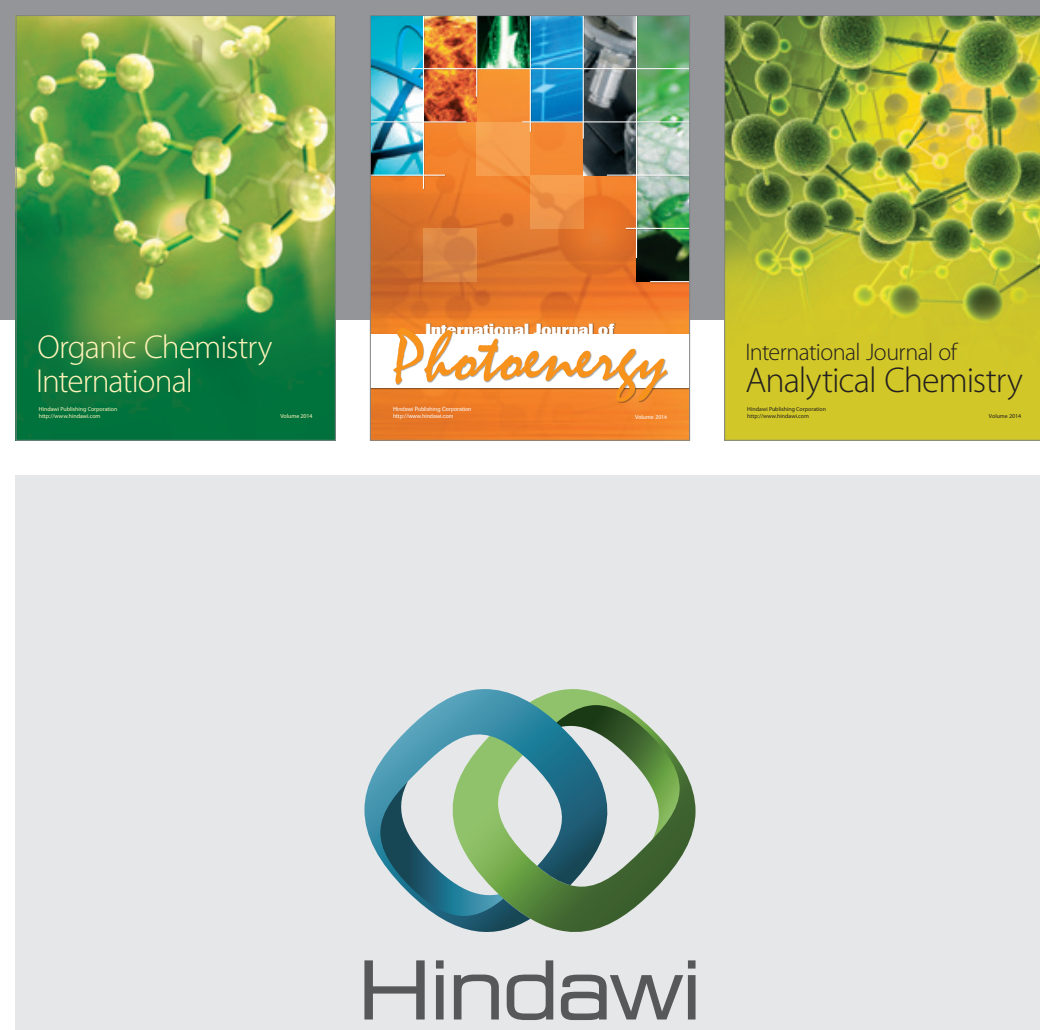

Submit your manuscripts at

http://www.hindawi.com
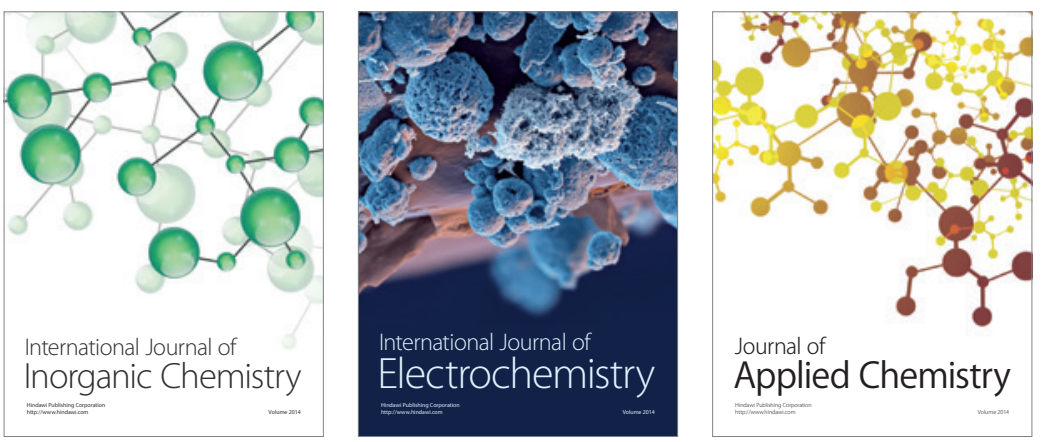

Journal of

Applied Chemistry
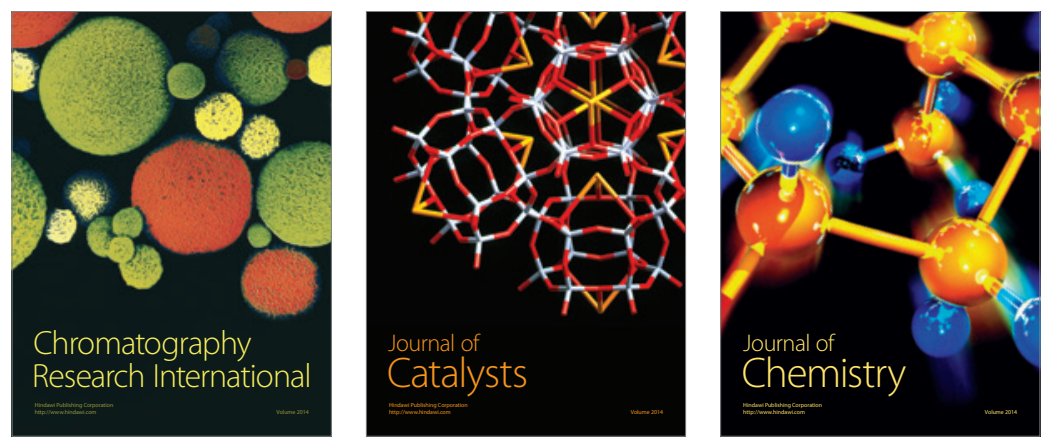
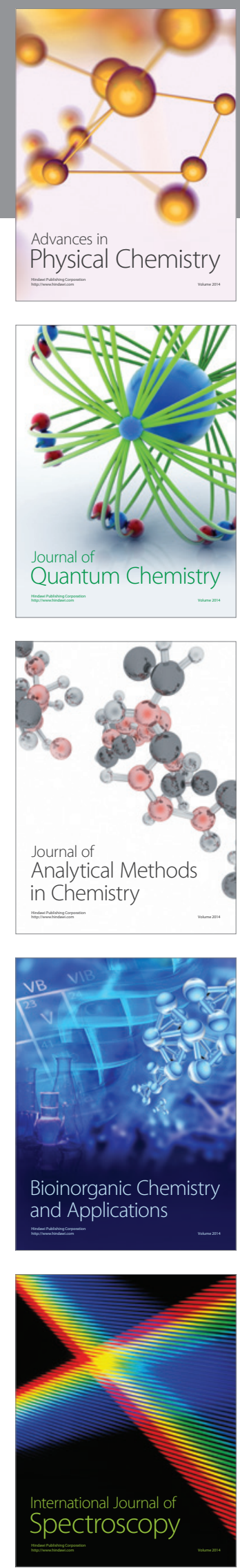